Voix et Images

\title{
D’Alain Grandbois à Madeleine Gagnon en passant par Rina Lasnier
}

\section{André Brochu}

Volume 37, numéro 3 (111), printemps-été 2012

URI : https://id.erudit.org/iderudit/1011963ar

DOI : https://doi.org/10.7202/1011963ar

Aller au sommaire du numéro

Éditeur(s)

Université du Québec à Montréal

ISSN

0318-9201 (imprimé)

1705-933X (numérique)

Découvrir la revue

Citer ce compte rendu

Brochu, A. (2012). Compte rendu de [D’Alain Grandbois à Madeleine Gagnon en passant par Rina Lasnier]. Voix et Images, 37(3), 179-184.

https://doi.org/10.7202/1011963ar d'utilisation que vous pouvez consulter en ligne.

https://apropos.erudit.org/fr/usagers/politique-dutilisation/ 


\author{
P O ÉS I E \\ D'Alain Grandbois à Madeleine Gagnon \\ en passant par Rina Lasnier \\ $+++$
}

ANDRÉ BROCHU

Université de Montréal

D'abord, un bref rappel.

La Bibliothèque du Nouveau Monde a publié, voilà plusieurs années déjà, l'édition critique des œuvres d'Alain Grandbois, notamment de ses œuvres poétiques ${ }^{1}$ qui, comme on sait, font l'essentiel de sa réputation. Dans le premier tome de Poésie, on reprenait les trois grands recueils (Les îles de la nuit, Rivages de l'homme et L'étoile pourpre) déjà parus et réédités, notamment, par l'Hexagone ${ }^{2}$, mais aussi des recueils inachevés ou inédits et des groupes de poèmes publiés en revue ou en cahiers. Le deuxième tome comprend une masse impressionnante de poèmes inédits, non revus pour la publication.

On peut juger regrettable que les trois recueils qui ont fait la renommée du poète se trouvent mêlés à des suites non approuvées par lui (Les îles de la nuit est suivi de Vent de nuit; Rivages de l'homme, de Passage de l'homme, etc.) et qui sont souvent loin de les valoir; et que Suite canadienne, par exemple, adjoigne à tout cela ses médiocres accents épiques mijotés à la sauce nationale-fédéraliste:

Je veux célébrer à mon tour mon pays,

Mon pays de l'Atlantique au Pacifique,

Des falaises froides de Terre-Neuve aux plages dorées de Victoria,

[...] Je veux célébrer les vertus d'un grand pays qui se nomme le Canada,

Les grands hommes, latins ou saxons, qui l'ont parcouru jadis parmi les dangers les

plus mortels,

Et ceux de ce jour qui voyagent à bord du Canadien Pacifique,

[...] Je veux célébrer mon pays, notre pays, votre pays,

Le Canada,

Où le soleil ne se couche jamais (Poésie, t. I, 372-373).

1 Alain Grandbois, Poésie, édition critique par Marielle Saint-Amour et Jo-Ann Stanton sous la direction de Ghislaine Legendre, Montréal, Presses de l'Université de Montréal, coll. «Bibliothèque du Nouveau Monde», 1990, t. I : 574 p. et t. II : 642 p. 2 Alain Grandbois, Poèmes. Les îles de la nuit, Rivages de l'homme, L'étoile pourpre, Poèmes épars, Montréal, l’Hexagone, coll. «Rétrospectives», 1979, 258 p. 
Ce dernier vers, qui gratifie le pays de dimensions qu'on attribue plutôt à l'ancien Empire britannique, n'est pas sans soulever l'étonnement...

Tout ceci pour donner, en guise d'introduction, un aperçu d'une entreprise d'édition intégrale de Grandbois sans doute louable, mais qui aurait pu isoler plus nettement les textes achevés de ceux auxquels le poète n'avait pas donné son aval. Sa gloire en eût été mieux servie.

\section{ATELIER PORTATIF}

Voici que paraît Les carnets d'Alain Grandbois ou l'atelier portatif d'un poète voyageur, de Jacinthe Martel et de Marie Pier Jolicœur ${ }^{3}$. Il ne s'agit pas d'une édition intégrale, comme le titre pourrait le donner à penser, mais d'une description très précise, voire technique (du point de vue de la critique génétique), de cinquante-huit petits cahiers (plus quelques ajouts) où l'écrivain a consigné, de façon très libre, les jaillissements de son inspiration de même qu'une foule de renseignements souvent étrangers à la littérature. Rien de plus désordonné que ces carnets non datés et utilisés de façon partielle, quand ce n'est cavalière (certains textes s'étalent uniquement sur les rectos, ou les versos, ou sur les pages du milieu alors que les autres restent blanches, etc.). Bref, Alain Grandbois s'en servait à des fins très particulières, et les publier tels quels serait non seulement inutile, mais impossible. Les deux auteures de la monographie font œuvre d'archivistes et complètent par là, sur un élément considérable du fonds Grandbois conservé à Bibliothèque et Archives nationales du Québec, le travail amorcé par les auteurs de l'édition critique (BNM), dont j'ai parlé plus haut. Celle-ci sert d'ailleurs de référence constante, et le livre sur les Carnets se présente un peu comme son appendice.

La poésie y occupe une belle place, et l'on y trouve le premier jet de nombreux textes des recueils publiés, en particulier L'étoile pourpre, ou d'échantillons de poèmes inédits (Poésie, t. II). Et voilà l'apport principal de cette publication, pour ceux que la poésie de Grandbois intéresse d'abord et avant tout. Une quarantaine de fac-similés, accompagnés de leur transcription, nous donnent accès à la version originale ou presque, manuscrite ou parfois tapuscrite, de dix poèmes d'assez grande dimension et permet d'apprécier, dès l'ébauche des textes, tout un travail de correction et de transformation. Certes, l'édition critique (Poésie, t. I et t. II) avait indiqué soigneusement les variantes, mais de façon beaucoup plus aride. On est ici placé directement devant l'élan créateur, dans son surgissement originaire, avec ses réussites et ses maladresses mêlées. Tel est bien l'atelier que nous promettent les auteures, avec ce qu'il comporte de fantaisie nomade, car le poète parcourt le vaste monde et les carnets lui permettent justement une consignation commode de l'inspiration. La formule de l'atelier avait déjà été appliquée à Francis Ponge dans l'édition critique de la Pléiade à laquelle a collaboré

$$
++
$$

3 Jacinthe Martel et Marie Pier Jolicœur, Les carnets d'Alain Grandbois ou l'atelier portatif d'un poète voyageur, Québec, Presses de l’Université Laval, coll. «L'archive littéraire au Québec. Série Approches», 2011, 188 p. 
Jacinthe Martel ${ }^{4}$, mais aussi et d'abord dans un mémorable ouvrage consacré à René Char ${ }^{5}$.

L'atelier ajoute une dimension visuelle, voire spectaculaire, à ce qui, sans doute, n'en a pas besoin. La poésie se suffit à elle-même. Pourtant, il n'est pas défendu à l'amateur de littérature de nourrir sa religion du texte par la contemplation des traces du surgissement, tout comme le croyant se repaît des aspects tangibles de la vérité révélée.

\section{L'ÂME FAITE CHAIR}

Ce n'est pas le premier choix de poèmes qu'on ait consacré à Rina Lasnier. Il y eut d'abord, en 1964, celui de Jean Marcel; puis, en 1970, un livre signé de l'auteure et intitulé La part du feu; et enfin, en 1981, Le choix de Rina Lasnier dans l'œuvre de Rina Lasnier ${ }^{6}$. Mais voici une anthologie pour le lecteur d'aujourd'hui ${ }^{7}$, et elle tient un compte généreux des derniers recueils, tout en accordant la place qui leur revient aux grandes réussites des années 1955-1970, et même aux recueils qui les ont préparées. Jocelyne Felx, qui a réalisé cette sélection, est elle-même poète et se révèle en très juste harmonie avec l'œuvre qu'elle présente, par la hauteur de la conception qu'elle se fait de la poésie et la connaissance du vaste intertexte dont Rina Lasnier s'est nourrie.

Mais qu'en est-il de cette rigoureuse demoiselle (Rina Lasnier, bien sûr!) qui reprend le flambeau des mains de poétesses apparues au cours des années 1930? Il y avait entre autres Jovette-Alice Bernier et Medjé Vézina, qui ont fait entendre des voix de femmes passablement libérées des contraintes de la tradition, notamment religieuse. Rina Lasnier peut sembler revenir sur le chemin parcouru, elle qui affirme avec vigueur sa foi chrétienne et une vision du monde entièrement imprégnée par l'amour du Christ. On s'étonnera qu'une telle ferveur ait pu inspirer l'un des accomplissements les plus remarquables de la poésie moderne du Québec. Et je dis bien «moderne», car le langage qui étale ses richesses d'un poème à l'autre tout au long de cette œuvre est étranger aux clichés de la tradition, et va puiser dans le fonds religieux les éclats susceptibles d'embellir et d'honorer les réalités les plus immédiates, les plus concrètes de l'existence. Comment concilier une spiritualité si orthodoxe, si passionnée, avec une telle attention aux vérités matérielles, à la nature, à l'amour charnel même - mais délesté, on le comprendra, de toute impureté et converti en totale lumière? Peu

$$
++
$$

4 Francis Ponge, Fuvres complètes, t. I, sous la direction de Bernard Beugnot, avec la collaboration de Michel Collot, Gérard Farasse, Jean-Marie Gleize, Jacinthe Martel, Robert Melançon et Bernard Veck, Paris, Gallimard, coll. «Bibliothèque de la Pléiade», 1999. Dans l'atelier du «Parti pris des choses» (57-69), etc. 5 René Char, Dans l'atelier du poète, Paris, Gallimard, coll. «Quarto», 1996, 1003 p. 6 Rina Lasnier, Rina Lasnier, textes choisis et présentés par Jean Marcel, Montréal, Fides, coll. «Classiques canadiens », 1964, 96 p. ; Rina Lasnier, La part du feu, Montréal, Éditions du Songe, coll. «Poésie du Québec», 1970, 91 p. ; Rina Lasnier, Le choix de Rina Lasnier dans l'œuvre de Rina Lasnier, Québec, Les Presses laurentiennes, coll. «Le choix de», 1981, 78 p. 7 Rina Lasnier, L'épanouissement de l'ombre. Poèmes choisis, choix et présentation de Jocelyne Felx, Montréal, Éditions du Noroît, coll. «Ovale», 2011, 173 p. 
d'écrivains, surtout dans notre jeune littérature, ont su allier l'humain et le divin de façon aussi étonnante. On aboutit à tout moment à des expressions troublantes de beauté, où les postulations naturelles et surnaturelles (celles-ci, pas toujours explicites) se fondent comme dans le bref poème suivant, tiré d'un des derniers recueils :

\author{
DOUCIS-TOI \\ Doucis-toi, doucis-toi... \\ défeuille-toi de turbulence \\ nudité d'excessive présence, \\ reprends ta robe ombrageante \\ doucie de fragile innocence... (161)
}

La nudité féminine semble être le sujet, au fait assez inattendu, de ces quelques vers. Elle est assimilée à une « turbulence » qu'il faudrait vaincre en «doucissant» (polissant) le corps, la personne. Se doucir et dompter ainsi la turbulence, c'est se "défeuiller " de celle-ci, et le mot n'est pas innocent puisqu'il évoque le travail de l'effeuilleuse pour réaliser justement la nudité. Ici, la nudité est ce dont on se dépouille dans un geste tout contraire, elle est l'« excessive présence» de la chair sur laquelle la robe jettera son ombre bénéfique. La robe est précisément ce qui « doucit» par son apport de la «fragile innocence».

À qui s'adresse la poète? À elle-même ou à une femme d'un autre monde, par exemple à une travailleuse du sexe? Elle s'adresse à toute femme dont l'innocence est menacée, et qui se doit de "doucir", c'est-à-dire devenir la douceur même, à toute force, sans le «a-» qui adoucit la douceur, fait d'elle une vertu ouateuse.

On pourrait dire que toute la démarche de Rina Lasnier consiste à renouveler, par la poésie, la vision des choses, notamment des réalités spirituelles et des figures célestes, mais aussi des fondements de la matière et du monde, en conciliant les inconciliables de la pensée traditionnelle, voire conservatrice. Ainsi en va-t-il de «La Malemer » (88-98), ce lieu sombre des origines qui est traversé et transformé par le Verbe divin et dont le salut s'accomplit en conformité avec l'acte du poème. Or, cet acte ne fait qu'un avec celui de l'amour humain, vécu dans la plus entière proximité des corps:

Comme l'amante endormie dans l'ardente captivité - immobile dans la pourpre muette de l'amant,

fluente et nocturne à la base du désir - obscurcie de sommeil et travestie d'innocence,

ses cheveux ouverts à la confidence - telles les algues du songe dans la mer écoutante,

la femme omniprésente dans la fabulation de la chair - la femme fugitive dans la fabulation de la mort (93). 
Chair et mort sont aux antipodes, c'est dire que la chair est vie, et la femme s'accomplit par une docilité à la matière ardente, bien entendu «doucie» et « défeuillée» de turbulence...

On voit que Rina Lasnier, figure majeure de nos lettres, n'a pas démérité de ces pionnières qui, au cours des années 1930, ont donné une voix à la femme et au désir. Sans doute a-t-elle pris le risque de réinventer Dieu, la Vierge, le Christ et maint motif biblique, mais elle l'a fait en donnant à la poésie des métaphores, une syntaxe et une métrique d'une singularité inconnue jusque-là.

\section{LA PENSÉE POÉSIE}

Madeleine Gagnon n'est pas la seule écrivaine à concilier des discours littéraires différents, et même opposés. Je pense, en l'occurrence, à ceux de poète, de romancière, d'essayiste (dans le style universitaire). Depuis que nos écrivains sont en grande partie diplômés des Facultés de lettres, on rencontre souvent des auteurs polyvalents, capables de disserter sur la théorie littéraire aussi bien que d'ajouter au trésor de la fiction.

Madeleine Gagnon, cependant, fait exception par la faculté très rare qu'elle a d'investir la théorie la plus avancée, voire la plus austère, par une bonne dose de poésie, et même de narration. C'est le cas, notamment, dans le recueil de textes divers - communications savantes, articles, témoignages, tous porteurs d'une réflexion sur le poème - qu'elle a publié récemment. On observe cette union des genres dès le texte qui donne son titre au livre, Donner ma langue au chant ${ }^{8}-$ formule où le jeu de mots s'efface vite devant une importante vérité. Car si donner sa langue au chat, selon l'expression bien connue, veut dire capituler devant une énigme, la donner au chant c'est faire, de cette énigme, un programme de recherche indéfinie, celle-là même qui fonde la nécessité de la poésie. La poésie est recherche de soi, du langage et de ce que le langage peut connaître, voire créer du monde même ${ }^{9}$.

Or il est impossible de méditer sur le chant sans introduire, dans l'appareil de l'investigation, le chant lui-même sous la forme de métaphores novatrices, tendues vers la captation du rêve, de l'imaginaire qui constitue la meilleure part du réel (absolu). Voici un exemple de théorie-poème consacrée précisément à la définition du poétique:

Le poème est cette effraction de l'être dans le brouillard du rêve de l'autre (être, chose) et dans la nuit des temps. Comme la parole de la séance (la parole pleine), il n'a ni commencement, ni fin (il est interminable), il s'écrit brisé, fracturé. Il est ce fragment ravi au silence éternel des dieux et permet cet envol outre-frontières vers une contrée sans limites où tous les poètes du monde ont aligné ces notes de

8 Madeleine Gagnon, Donner ma langue au chant, Montréal, Éditions du Noroît, coll. «Chemins de traverse », 2011, 162 p. 9 Pour un aperçu de la poésie de Madeleine Gagnon, réunie en un seul volume, on lira mon compte rendu: «Deux rétrospectives. Madeleine Gagnon, Alexis Lefrançois», Voix et Images, vol. XXXIII, n 1 , automne 2007, p. 158-163. 
l'autre-rêvé qui flottent au hasard du silence des ans et qui se captent au toucher de l'écoute incertaine (62).

Nous avons affaire ici à un discours théorique qui, loin de proposer une explication rationnelle et simpliste des choses, demande à être lui-même déchiffré à la lumière de ce qu'il s'attache à commenter.

Et c'est le mouvement spontané de la réflexion menée par Madeleine Gagnon. D'abord est posée l'énigme du monde et de la poésie qui en rend compte, et le langage se fait mi-clair, mi-obscur ; mais cela, pour arriver le plus souvent - ce n'est pas toujours le cas - à des propositions transparentes et accessibles à l'entendement «commun ». À cette fin, un récit comme celui de l'attirant jeune homme rencontré dans un train et qui, inopinément, se suicide (16-17), ou encore, quelque trait plus immédiat d'autobiographie - puisque « toute écriture de fiction (poésie ou prose) et, en grande partie l'écriture de l'essai, est bel et bien autobiographique » (47) - , favorisent mieux que tout l'établissement d'une intelligibilité.

La théorie, chez Madeleine Gagnon, concerne aussi bien la psychanalyse que la philosophie, et bien d'autres sciences humaines. Elle se réclame de nombreux penseurs, tels Jacques Lacan, René Major, Jacques Derrida, Emmanuel Kant, Paul Ricœur, René Thom, Betty Friedan, Luce Irigaray; ou encore, en poésie proprement dite, Charles Baudelaire, Paul Claudel, René Char, Francis Ponge, Edmond Jabès, Louis Aragon, Élias Canetti... Chacun apporte sa contribution à la connaissance de l'énigme des êtres et des choses, par l'intuition plus que par l'élucidation, la poésie ne gagnant rien à être traduite en évidences.

La poésie, écrit encore Madeleine Gagnon avec une belle simplicité, «c'est la pensée qui chante» (63), et il n'est pas étonnant que la pensée qui en rend compte ait sa composante de chant elle aussi. Dès lors, poésie et pensée se mirent l'une l'autre à l'infini. Mais pourquoi en va-t-il ainsi?

Un texte répond de façon précise à cette question. L'obscurité dans laquelle se déploie, de façon très nécessaire, la pensée-poème de Madeleine Gagnon est justifiée par la condition même de la femme (99-100), laquelle pousse à explorer les «traces d'avant la loi signifiante» (99). La loi signifiante, ou logos, est la chasse gardée de l'homme. En regard de cette loi, affirme un Jacques Lacan, «la Femme n'existe pas» (98). La femme qui écrit est donc condamnée à vivre la pénombre du sens, le mystère du monde, et la poésie est la voie privilégiée pour le faire.

On voit l'ampleur des considérations rassemblées autour de la question, double et une, de la femme et de l'écriture, tout à fait centrale dans l'œuvre de Madeleine Gagnon. 